\title{
A New Globally Convergent of Spectral Hideaki - Yasushi Type Conjugate Gradient Method
}

\author{
Sawsan S. Ismael \\ Department of Mathematics, College of Education of pure science \\ University of Mosul, Iraq

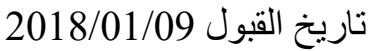

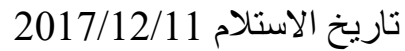

\section{Abstract}

This paper presents two new spectral conjugate gradient methods which are designed for solving nonlinear unconstrained optimization problems. These methods are based on the idea of the Hideaki - Yasushi method. Which produce sufficient descent search direction at every iteration. Experimental results indicate that the new proposed methods more efficient than the Hideaki - Yasush method.

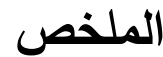

في هذا البحث تم اســـتحداث طريقتين جديدتين من طرائق التدرج المترافق الطيفية لحل مســـائل الأمثلية اللاخطية وغير المقيدة. هاتان الطريقتان معتمدتان في في فئني الأســـاس على فكرة طريقة Hideaki - Yasush. وقد أثبتت الطريقتان إن لهما اتجاه بحث ذو انحدار شـديد عند كل تكرار ـ النتائج العددية أثتتت كفاءة الطريقتين

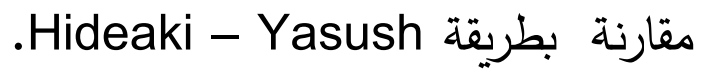

\section{Introduction}

The nonlinear conjugate gradient (CG) method is designed to solve the following unconstrained optimization problem

$$
\min \left\{f(x) \mid x \in R^{n}\right\}
$$

where $f: R^{n} \rightarrow R$ is a continuously differentiable nonlinear function whose gradient is denoted by $g$. Due to its simplicity and its very low memory requirement, the $\mathrm{CG}$ method has played a special rule for solving 
large scale nonlinear optimization problems. The iterative formula of the CG method is given by

$$
x_{k+1}=x_{k}+\alpha_{k} d_{k}
$$

where $\alpha_{k}>0$ is a step length which is computed by carrying out a line search and satisfies the standard Wolfe (SW ) conditions

$$
\begin{gathered}
f\left(x_{k}+\alpha_{k} d_{k}\right) \leq f\left(x_{k}\right)+\delta_{1} \alpha_{k} d_{k}^{T} g_{k} \\
g\left(x_{k}+\alpha_{k} d_{k}\right)^{T} d_{k} \geq \delta_{2} d_{k}^{T} g_{k}
\end{gathered}
$$

with $0<\delta_{1}<\delta_{2}<1$, and $d_{k+1}$ is the search direction defined by

$$
d_{k+1}= \begin{cases}-g_{1} & k=1, \\ -g_{k+1}+\beta_{k} d_{k} & k>1,\end{cases}
$$

The search direction $d_{k}$ is generally required to satisfy :

$$
g_{k+1}^{T} d_{k+1}<0,
$$

which guarantees that $d_{k}$ is a descent direction of $f(x)$ at $x_{k}$. In order to guarantee the global convergence, we sometimes require $d_{k}$ to satisfy a sufficient descent condition :

$$
g_{k+1}^{T} d_{k+1} \leq-c\left\|g_{k+1}\right\|^{2}
$$

where $c$ is a constant [8]. Different CG methods correspond to different choices for the scalar $\beta_{k}$. The well known formulas for $\beta_{k}$ such as $\beta_{k}^{H S}$ ( Hestenes, Stiefel [7]), $\beta_{k}^{F R}$ (Fletcher, Reeves [6]), $\beta_{k}^{P R P}$ ( Polak, Ribiere and Polyak [9]), $\beta_{k}^{C D}$ (Fletcher [5]), $\beta_{k}^{L S}$ ( Liu, Storey [8]), $\beta_{k}^{D Y}$ (Dai, Yuan [4]) can be found in many related literatures. Recently, Hideaki and Yasushi [14] proposed a new conjugate gradient method which was obtained by modifying the DY method and called HY method. A nice property of the HY method is that it generates sufficient descent directions. The parameter $\beta_{k}$ in HY method is given by

$$
\beta_{k}^{H Y}=\frac{g_{k+1}^{T} g_{k+1}}{\frac{2}{\alpha_{k}}\left(f_{k}-f_{k+1}\right)}
$$

performs more effective more details can be found in [14]. It is well know that the linear conjugate gradient methods generate a sequence of search directions $d_{k+1}$ such that the following conjugacy condition holds : 


$$
d_{i}^{T} H d_{j}=0, \forall i \neq j,
$$

where $H$ is the Hessian of the objective function. For general nonlinear function $f$, we know by the mean value theorem that there exists some $t \in(0,1)$ such that

$$
d_{k+1}^{T} y_{k}=\alpha_{k} d_{k+1}^{T} \nabla^{2} f\left(x_{k}+t \alpha_{k} d_{k}\right) .
$$

Therefore, it is reasonable to replace (10) with the following conjugacy condition :

$$
d_{k+1}^{T} y_{k}=0 .
$$

Recently, extensions of (10) have been studied in [4,7] that are based on the standard secant equation

$$
H_{k+1} y_{k}=v_{k} .
$$

from (12) and the search direction $d_{k+1}$ can be calculated in the form

$$
d_{k+1}=-H_{k+1} g_{k+1}
$$

we have

$$
d_{k+1}^{T} y_{k}=-\left(H_{k+1} g_{k+1}\right)^{T} y_{k}=-g_{k+1}^{T} H_{k+1} y_{k}=-g_{k+1}^{T} v_{k} .
$$

By introducing a scaling factor $t$, Dai and Liao considered a generalized conjugate condition,

$$
d_{k+1}^{T} y_{k}=-\operatorname{tg}_{k+1}^{T} v_{k}, t \geq 0,
$$

where $t$ is a parameter. In the case $t=0$, then (15) becomes (11). In case $t=1$, (15) reduced to (14). Furthermore, if exact line search is used, then $g_{k+1}^{T} v_{k}=0$ holds for all $k$. It follows that both (14) and (15) coincide with (9). More details can be found in [10]

Another popular method to solving problem (1) is the spectral gradient method, which was developed originally by Barzilai and Borwein in 1988. The direction $d_{k+1}$ is given by the following way

$$
d_{k+1}=-\theta_{k} g_{k+1}+\beta_{k} d_{k}
$$

where $\theta_{k}$ is scalar parameter which follows to be determined. More details can be found in [3]. 
The structure of the paper is as follows. In section (2) we present the new spectral conjugate gradient methods and descent algorithm. Section (3) show that the search direction generated by this proposed algorithm at each iteration satisfies the sufficient descent condition. Section (4) establishes the global convergence property for the new CGmethod. Section (5) establishes some numerical results to show the effectiveness of the proposed CG-method and section (6) gives a brief conclusions and discussions.

\section{A New Spectral Conjugate Gradient Methods}

In this article we present a modification of the Hideaki and Yasushi rule, is defined on the basis of $\beta_{k}^{H Y}$ as follows :

$$
\beta_{k}^{S A H}=\frac{g_{k+1}^{T} g_{k+1}}{\frac{2}{\alpha_{k}}\left(f_{k}-f_{k+1}\right)}-\frac{g_{k+1}^{T} g_{k+1}\left(v_{k}^{T} g_{k+1}\right)}{\left(\frac{2}{\alpha_{k}}\left(f_{k}-f_{k+1}\right)\right)^{2}}
$$

Observe that

$$
\beta_{k}^{S A H}=\frac{g_{k+1}^{T} g_{k+1}}{\frac{2}{\alpha_{k}}\left(f_{k}-f_{k+1}\right)}\left[1-\frac{v_{k}^{T} g_{k+1}}{\frac{2}{\alpha_{k}}\left(f_{k}-f_{k+1}\right)}\right]=\beta_{k}^{H Y} t_{k}
$$

where

$$
t_{k}=1-\frac{v_{k}^{T} g_{k+1}}{\frac{2}{\alpha_{k}}\left(f_{k}-f_{k+1}\right)}
$$

From the second Wolfe condition it follows that $v_{k}^{T} g_{k+1} \geq \delta_{2} v_{k}^{T} g_{k}=\delta_{2} \alpha_{k} d_{k}^{T} g_{k}$. In [14] Hideaki and Yasushi proved the $\frac{2}{\alpha_{k}}\left(f_{k}-f_{k+1}\right) \geq-2 \delta_{1} g_{k}^{T} d_{k}$. It follows that $\frac{v_{k}^{T} g_{k+1}}{\frac{2}{\alpha_{k}}\left(f_{k}-f_{k+1}\right)} \geq \frac{\delta_{2} \alpha_{k} d_{k}^{T} g_{k}}{-2 \delta_{1} g_{k}^{T} d_{k}}=\frac{\delta_{2} \alpha_{k}}{-2 \delta_{1}}$. Hence $t_{k}=1-\frac{v_{k}^{T} g_{k+1}}{\frac{2}{\alpha_{k}}\left(f_{k}-f_{k+1}\right)} \leq 1-\left[\frac{\delta_{2} \alpha_{k}}{-2 \delta_{1}}\right]=1+\frac{\delta_{2} \alpha_{k}}{2 \delta_{1}}=Z_{k}$ Therefore, $\beta_{k}^{S A H} \leq \beta_{k}^{H Y} Z_{k}$.

To determine the parameter $\theta_{k}$ in $d_{k+1}=-\theta_{k} g_{k+1}+\beta_{k} v_{k} \quad$ we suggest the following two procedures, in order to satisfy both the descent condition and the conjugate condition in the frame of conjugate gradient methods : 
1-The first procedure is based on the descent condition. Putting $\beta_{k}^{S A H}$ into (16) with descent condition, we obtain :

$$
\begin{aligned}
& d_{k+1}^{T} g_{k+1}=-\theta_{k} g_{k+1}^{T} g_{k+1}+\beta_{k}^{S A H} v_{k}^{T} g_{k+1}<0 \\
= & -\theta_{k} g_{k+1}^{T} g_{k+1}+\left[\frac{g_{k+1}^{T} g_{k+1}}{\frac{2}{\alpha_{k}}\left(f_{k}-f_{k+1}\right)}-\frac{g_{k+1}^{T} g_{k+1}\left(v_{k}^{T} g_{k+1}\right)}{\left(\frac{2}{\alpha_{k}}\left(f_{k}-f_{k+1}\right)\right)^{2}}\right] v_{k}^{T} g_{k+1}<0 \\
& {\left[\frac{g_{k+1}^{T} g_{k+1}}{\frac{2}{\alpha_{k}}\left(f_{k}-f_{k+1}\right)}-\frac{g_{k+1}^{T} g_{k+1}\left(v_{k}^{T} g_{k+1}\right)}{\left(\frac{2}{\alpha_{k}}\left(f_{k}-f_{k+1}\right)\right)^{2}} v_{k}^{T} g_{k+1}<\theta_{k} g_{k+1}^{T} g_{k+1}\right.}
\end{aligned}
$$

From (18) we get :

$$
\theta_{k}^{S 1}>\left[\frac{g_{k+1}^{T} g_{k+1}}{\frac{2}{\alpha_{k}}\left(f_{k}-f_{k+1}\right)}-\frac{g_{k+1}^{T} g_{k+1}\left(v_{k}^{T} g_{k+1}\right)}{\left(\frac{2}{\alpha_{k}}\left(f_{k}-f_{k+1}\right)\right)^{2}}\right] \frac{v_{k}^{T} g_{k+1}}{g_{k+1}^{T} g_{k+1}}
$$

2- The second procedure is based on the conjugate condition. Substituting (16) into (14), we obtain :

$$
\begin{aligned}
& d_{k+1}^{T} y_{k}=-\theta_{k} g_{k+1}^{T} y_{k}+\beta_{k}^{S A H} v_{k}^{T} y_{k} \\
& d_{k+1}^{T} y_{k}=-\theta_{k} g_{k+1}^{T} y_{k}+\left[\frac{g_{k+1}^{T} g_{k+1}}{\frac{2}{\alpha_{k}}\left(f_{k}-f_{k+1}\right)}-\frac{g_{k+1}^{T} g_{k+1}\left(v_{k}^{T} g_{k+1}\right)}{\left(\frac{2}{\alpha_{k}}\left(f_{k}-f_{k+1}\right)\right)^{2}} v_{k}^{T} y_{k}\right. \\
& \theta_{k} g_{k+1}^{T} y_{k}=\left[\frac{g_{k+1}^{T} g_{k+1}}{\frac{2}{\alpha_{k}}\left(f_{k}-f_{k+1}\right)}-\frac{g_{k+1}^{T} g_{k+1}\left(v_{k}^{T} g_{k+1}\right)}{\left(\frac{2}{\alpha_{k}}\left(f_{k}-f_{k+1}\right)\right)^{2}} v_{k}^{T} y_{k}+g_{k+1}^{T} v_{k}\right.
\end{aligned}
$$

we have : 


$$
\theta_{k}^{S 2}=\left[\frac{g_{k+1}^{T} g_{k+1}}{\frac{2}{\alpha_{k}}\left(f_{k}-f_{k+1}\right)}-\frac{g_{k+1}^{T} g_{k+1}\left(v_{k}^{T} g_{k+1}\right)}{\left(\frac{2}{\alpha_{k}}\left(f_{k}-f_{k+1}\right)\right)^{2}}\right] \frac{v_{k}^{T} y_{k}}{g_{k+1}^{T} y_{k}}+\frac{g_{k+1}^{T} v_{k}}{g_{k+1}^{T} y_{k}}
$$

As above, since $g_{k+1}^{T} v_{k} \rightarrow 0$ along the iterations, $\theta_{k}^{S 1}$ obtained from the Newton direction paradigm is very similar to $\theta_{k}^{S 2}$ based on the conjugacy condition.

Now we can obtain the new conjugate gradient algorithms.

\section{New Algorithm :}

Step 1. Select $x_{1} \in R^{n}$ and the parameters $0<\delta_{1}<\delta_{2}<1$. Compute $f\left(x_{1}\right)$ and $g_{1}$. Consider $d_{1}=-g_{1}$ and set the initial guess $\alpha_{1}=1 /\left\|g_{1}\right\|$.

Step 2. Test for continuation of iterations. If $\left\|g_{k+1}\right\| \leq 10^{-6}$, then stop.

Step 3. Line search. Compute $\alpha_{k+1}>0$ satisfying the Wolfe line search condition (3) and (4) and update the variables $x_{k+1}=x_{k}+\alpha_{k} d_{k}$.

Step 4. $\beta_{k}$ conjugate gradient parameter which defined in (17) .

Step 5. $\theta_{k}$ is computed as in (19) and (21) where $\theta_{k}>1 / 4$.

Step 6. Direction computation. Compute $d_{k+1}=-\theta_{k} g_{k+1}+\beta_{k} v_{k}$. If the restart criterion of Powell $\left|g_{k+1}^{T} g_{k}\right| \geq 0.2\left\|g_{k+1}\right\|^{2}$, is satisfied, then set $d_{k+1}=-\theta_{k} g_{k+1}$ , else set $k=k+1$ and continue with step2 .

\section{The sufficient descent condition}

In this section we shall introduce the new theorem which is ensure the sufficient descent of the new methods given in (17) with (19) and (21).

\section{Theorem (3.1)}

If $\theta_{k}^{S 1}>1 / 4$, then the direction $d_{k+1}=-\theta_{k}^{S 1} g_{k+1}+\beta_{k}^{S A H} v_{k}$ satisfies the sufficient descent direction.

$$
g_{k+1}^{T} d_{k+1} \leq-\left[\theta_{k}^{S 1}-\frac{1}{4}\right]\left\|g_{k+1}\right\|^{2} .
$$




\section{Proof.}

Since $d_{0}=-g_{0}$, we have $g_{0}^{T} d_{0} \leq-\left\|g_{0}\right\|^{2}<0$. Assume by induction that

$$
g_{k}^{T} d_{k} \leq-c\left\|g_{k}\right\|^{2}<0 \text { where } 0<c<1
$$

which is a sufficient descent direction. To complete the proof, we have to show that the theorem is true for all $k+1$. Multiplying (16) by $g_{k+1}^{T}$ we have :

$$
\begin{aligned}
& g_{k+1}^{T} d_{k+1}=-\theta_{k}^{S 1}\left\|g_{k+1}\right\|^{2}+\beta_{k}^{S A H} g_{k+1}^{T} v_{k} \\
& =-\theta_{k}^{S 1}\left\|g_{k+1}\right\|^{2}+\left[\frac{g_{k+1}^{T} g_{k+1}}{\frac{2}{\alpha_{k}}\left(f_{k}-f_{k+1}\right)}-\frac{g_{k+1}^{T} g_{k+1}\left(v_{k}^{T} g_{k+1}\right)}{\left(\frac{2}{\alpha_{k}}\left(f_{k}-f_{k+1}\right)\right)^{2}}\right] g_{k+1}^{T} v_{k}
\end{aligned}
$$

Now, using the inequality $u^{T} v \leq 1 / 2\left(\|u\|^{2}+\|v\|^{2}\right)$ to the second term of the right hand side of the above equality, with $u=\left(\left(2 / \alpha_{k}\right)\left(f_{k}-f_{k+1}\right)\right) g_{k+1}$ and $v=\left(g_{k+1}^{T} d_{k}\right) y_{k}$ we get :

$$
\begin{aligned}
\frac{g_{k+1}^{T} g_{k+1}\left(v_{k}^{T} g_{k+1}\right)}{\frac{2}{\alpha_{k}}\left(f_{k}-f_{k+1}\right)}= & \frac{\left[\left(\frac{1}{\alpha_{k}}\left\{2\left(f_{k}-f_{k+1}\right)\right\}\right) g_{k+1} / \sqrt{2}\right]^{T}\left[\sqrt{2}\left(v_{k}^{T} g_{k+1}\right) g_{k+1}\right]}{\left(\frac{2}{\alpha_{k}}\left(f_{k}-f_{k+1}\right)\right)^{2}} \\
& \leq \frac{\frac{1}{2}\left[\frac{1}{2}\left(\frac{1}{\alpha_{k}}\left\{2\left(f_{k}-f_{k+1}\right)\right\}\right)^{2}\left\|g_{k+1}\right\|^{2}+2\left(v_{k}^{T} g_{k+1}\right)^{2}\left\|g_{k+1}\right\|^{2}\right]}{\left(\frac{2}{\alpha_{k}}\left(f_{k}-f_{k+1}\right)\right)^{2}} \\
& =\frac{1}{4}\left\|g_{k+1}\right\|^{2}+\frac{\left(v_{k}^{T} g_{k+1}\right)^{2}\left\|g_{k+1}\right\|^{2}}{\left(\frac{2}{\alpha_{k}}\left(f_{k}-f_{k+1}\right)\right)^{2}}
\end{aligned}
$$

from (24) and (25) we have

$$
\begin{gathered}
g_{k+1}^{T} d_{k+1} \leq-\theta_{k}^{S 1}\left\|g_{k+1}\right\|^{2}+\frac{1}{4}\left\|g_{k+1}\right\|^{2} \\
g_{k+1}^{T} d_{k+1} \leq-\left[\theta_{k}^{S 1}-\frac{1}{4}\right]\left\|g_{k+1}\right\|^{2} .
\end{gathered}
$$


To conclude, the sufficient descent condition from (22), the quantity $\theta_{k}^{S 1}-1 / 4$ is required to be nonnegative. Supposing that $\theta_{k}^{S 1}-1 / 4>0$, then the direction given by (16) and (17) is a descent direction more details can be found in [2].

Remark : we use similarly technique to classical algorithm $\theta_{k}^{S 2}$.

\section{Convergence analysis}

In this section we analyze the convergence of the algorithm (2) and (16), where $\theta_{k}$ and $\beta_{k}$ are given by (17), (19) and (21) respectively. In the following we consider that

$$
g_{k+1} \neq 0, \forall k \geq 1 .
$$

Otherwise, a stationary point is at hand. We make the following basic assumptions on the objective function.

\section{Definition 4.1}

A twice continuously differentiable function $f$ is said to be uniformly convex on the nonempty open convex set $S$ if and only if there exists $M>0$ such that

$$
(g(x)-g(y))^{T} .(x-y) \geq M\|x-y\|^{2}, x, y \in S
$$

or, equivalently, there exists $r>0$ such that

$$
z^{T} \nabla^{2} f(x) z \geq r\|z\|^{2}, \forall x \in S, \forall z \in R^{n} .
$$

see [12].

\section{Assumption 1}

The level set $\quad l=\left\{x: f(x) \leq f\left(x_{1}\right)\right\}$ is bounded ; that is, there exists a constant $B>0$ such that

$$
\|d\| \leq B, \forall x \in l .
$$

\section{Assumption 2}

In some neighborhood $N$ of $l(l \subseteq N), f$ is continuously differentiable, and its gradient is Lipschitz continuous ; that is, there exists a constant $L>0$ such that

$$
\|\nabla f(x)-\nabla f(y)\| \leq L\|x-y\|, \forall x, y \in N .
$$


The following proposition is now immediate [12-13].

\section{Proposition 4.1}

Under Assumptions 1 and 2 on $f$, there exists a constant $\bar{\gamma}>0$ such that

$$
\|\nabla f(x)\| \leq \bar{\gamma}, \forall x \in l
$$

\section{Lemma 4.1.}

Suppose that Assumptions 1 and 2 hold. Consider any conjugate gradient method in the form (2)-(14), where $d_{k+1}$ is a descent direction and $\alpha_{k}$ is computed using the strong Wolfe line search conditions. If

$$
\sum_{k \geq 0} \frac{1}{\left\|d_{k+1}\right\|^{2}} \cdot=\infty
$$

then we have

$$
\liminf _{k \rightarrow \infty}\left\|g_{k}\right\|=0
$$

\section{Theorem 4.2}

Suppose that Assumptions 1 and 2 and the descent condition hold. Consider a conjugate gradient method in the form (17) - (19) with $\theta_{k}^{S 1}$ and $\beta_{k}^{S A H}$, where $\alpha_{k}$ is computed from the standard Wolfe line search conditions (3) - (4). Suppose that there exists the positive constant $\tau$ such that $1 / 4 \leq \theta_{k} \leq \tau$ for all $k \geq 1$. If the objective function is uniformly convex on $S$, then $\lim _{k \rightarrow \infty}\left\|g_{k}\right\|=0$.

\section{Proof :}

Now, from $\beta_{k}^{S A H} \leq \beta_{k}^{H Y} Z_{k}$ with $\theta_{k}^{S 1}$ it follows that $f$ is a uniformly convex function. Because the descent condition hold, we have $d_{k+1} \neq 0$. Also, from Assumptions 1 and 2, Proposition 4.1, Lemma 4.2, we have 


$$
\begin{aligned}
\left\|d_{k+1}\right\| & =\left\|-\theta_{k}^{S 1} g_{k+1}+\beta_{k}^{S A H} d_{k}\right\| \\
& \leq\left|\theta_{k}^{S 1}\right|\left\|g_{k+1}\right\|+\left|\beta_{k}^{H Y} Z_{k}\right|\left\|d_{k}\right\| \\
& \leq \tau\left\|g_{k+1}\right\|+\left(\frac{\left\|g_{k+1}\right\|^{2}}{\frac{2}{\alpha_{k}}\left(f_{k}-f_{k+1}\right)} Z_{k}\right)\left\|d_{k}\right\| \\
& \leq \tau \bar{\gamma}+Z_{k} \frac{[-\bar{\gamma}]^{2}}{2 c \delta_{1}\left\|g_{k}\right\|^{2}} B \\
\left\|d_{k+1}\right\| & \leq(\tau+C \bar{\gamma}) \bar{\gamma}, \quad C=Z_{k} \frac{B}{2 c \delta_{1}\left\|g_{k}\right\|^{2}}
\end{aligned}
$$

This relation shows that

$$
\sum_{k \geq 1} \frac{1}{\left\|d_{k+1}\right\|^{2}} \cdot \geq\left(\frac{1}{\left(c_{1}+C_{\gamma}^{-}\right) \bar{\gamma}}\right) \sum_{k \geq 1} 1=\infty .
$$

Therefore, from Lemma 4.1 we have $\liminf _{k \rightarrow \infty}\left\|g_{k}\right\|=0$, which for uniformly convex function is equivalent to $\lim _{k \rightarrow \infty}\left\|g_{k}\right\|=0$.

Remark : we use similarly technique to classical algorithm $\theta_{k}^{s 2}$.

\section{Numerical Results :}

In this section, we reported some numerical results obtained with the implementation of the new methods on a set of unconstrained optimization test problems taken from (Andrie, 2008) [1].

We selected (15) large scale unconstrained optimization test problems. For each test function we have considered 10 numerical experiments with number of variables $n=100,200, \ldots \ldots 1000$. We use $\delta_{1}=10^{-4}$ and $\delta_{2}=0.9$ in the line search routine (3) - (4). All these methods terminate when the following stopping criterion is met $\left\|g_{k+1}\right\| \leq 10^{-6}$.

All codes are written in double precision FORTRAN Language with F90 default compiler settings. We record the number of iterations calls (NOI), and the number of restart calls (IRS) for the purpose our comparisons. 
A New Globally Convergent of Spectral Hideaki - Yasushi Type Conjugate ...

Table (5.1) : Comparison of methods for $n=100$

\begin{tabular}{|c|c|c|c|c|c|c|}
\hline \multirow{3}{*}{$\begin{array}{c}\text { Test } \\
\text { problems } \\
\text { Extended Rosenbrock }\end{array}$} & \multicolumn{2}{|c|}{$\beta_{k}^{H Y}$} & \multicolumn{2}{|c|}{$\theta_{k}^{S 1}$} & \multicolumn{2}{|c|}{$\theta_{k}^{S 2}$} \\
\hline & NOI & IRS & NOI & & NOI & IRS \\
\hline & 20 & 13 & 20 & 13 & 20 & 12 \\
\hline Extended While \& Holst & 54 & 20 & 46 & 17 & 46 & 17 \\
\hline Extended PSC 1 & 34 & 17 & 34 & 16 & 33 & 17 \\
\hline Extended Maratos & 14 & 9 & 19 & 10 & 21 & 11 \\
\hline Quadratic QF2 & 48 & 16 & 36 & 12 & 45 & 15 \\
\hline Arwhead & 19 & 14 & 12 & 7 & 12 & 7 \\
\hline Nondia & 39 & 19 & 37 & 17 & 36 & 16 \\
\hline Partial Perturbed Quad. & 593 & 199 & 213 & 74 & 213 & 74 \\
\hline Liarwhd & 29 & 14 & 33 & 16 & 33 & 15 \\
\hline Denschnc & 23 & 21 & 22 & 15 & 20 & 16 \\
\hline Extended Block Diagonal & 36 & 17 & 37 & 15 & 35 & 14 \\
\hline Generalized Quad. GQ1 & 51 & 20 & 47 & 18 & 51 & 20 \\
\hline Sincos & 25 & 18 & 25 & 10 & 25 & 10 \\
\hline Liarwhd (CUTE) & $\mathrm{F}$ & $\mathrm{F}$ & 777 & 284 & 796 & 286 \\
\hline Generalized Quad. GQ2 & 162 & 60 & 159 & 57 & 137 & 52 \\
\hline Total & 1147 & 457 & 740 & 297 & 727 & 296 \\
\hline
\end{tabular}

Table (5.2) : Comparison of methods for $n=1000$

\begin{tabular}{|c|c|c|c|c|c|c|}
\hline \multirow{3}{*}{$\begin{array}{c}\text { Test } \\
\text { problems } \\
\text { Extended Rosenbrock }\end{array}$} & \multicolumn{2}{|c|}{$\beta_{k}^{H Y}$} & \multicolumn{2}{|c|}{$\theta_{k}^{S 1}$} & \multicolumn{2}{|c|}{$\theta_{k}^{S 2}$} \\
\hline & NOI & IRS & NOI & IRS & NOI & IRS \\
\hline & 39 & 21 & 33 & 21 & 33 & 21 \\
\hline Extended While \& Holst & 55 & 20 & 49 & 18 & 51 & 19 \\
\hline Extended PSC 1 & $\mathrm{~F}$ & $\mathrm{~F}$ & 237 & 200 & 301 & 263 \\
\hline Extended Maratos & 75 & 67 & 23 & 18 & 53 & 47 \\
\hline Quadratic QF2 & 51 & 17 & 42 & 14 & 51 & 17 \\
\hline Arwhead & 24 & 21 & 32 & 20 & 25 & 22 \\
\hline Nondia & 43 & 20 & 42 & 19 & 42 & 19 \\
\hline
\end{tabular}


A New Globally Convergent of Spectral Hideaki - Yasushi Type Conjugate ...

\begin{tabular}{|l|c|c|c|c|c|c|}
\hline Partial Perturbed Quad. & F & F & F & F & F & F \\
\hline Liarwhd & 98 & 85 & 64 & 50 & 36 & 25 \\
\hline Denschnc & 21 & 19 & 13 & 11 & 21 & 19 \\
\hline Extended Block Diagonal & 47 & 18 & 37 & 17 & 39 & 18 \\
\hline Generalized Quad. GQ1 & 52 & 21 & 52 & 21 & 50 & 18 \\
\hline Sincos & 38 & 19 & 35 & 14 & 33 & 14 \\
\hline Liarwhd (CUTE) & $\mathrm{F}$ & $\mathrm{F}$ & 1566 & 906 & 701 & 252 \\
\hline Generalized Quad. GQ2 & 1420 & 474 & 1427 & 479 & 1393 & 469 \\
\hline \multicolumn{1}{|c|}{ Total } & 1963 & $\mathbf{8 0 2}$ & $\mathbf{1 8 4 9}$ & $\mathbf{7 0 2}$ & $\mathbf{1 8 2 7}$ & $\mathbf{7 0 8}$ \\
\hline
\end{tabular}

\section{Conclusions}

Form the numerical results of the above tables, we say that the results of Table (5.1) and Table (5.2) give a general comparison between $\mathrm{HY}$ and two new spectral CG-methods taking non linear test function with $\mathrm{n}=100,1000$. This table indicates that the modified methods saves (16-17) $\%$ NOI and (20) \% IRS. The Percentage Performance of the improvements of the Table (5.1) and Table (5.2) are given by the following table (5.3).

Table(5.3): Relative efficiency of the new Algorithm

\begin{tabular}{|c||c|c|}
\hline Tools & NOI & IRS \\
\hline \hline M Dai-Yuan method & $100 \%$ & $100 \%$ \\
\hline \hline New Algorithm with $\theta_{k}^{S 1}$ & $83.24 \%$ & $79.34 \%$ \\
\hline \hline New Algorithm with $\theta_{k}^{S 2}$ & $82.12 \%$ & $79.74 \%$ \\
\hline
\end{tabular}




\section{References}

[1] Andrei N. (2008), An Unconstrained Optimization test function collection. Adv. Model. Optimization . 10. pp.147-161.

[2] Andrei N. (2010), New accelerated conjugate gradient algorithms as a modification of Dai_Yuan's computational scheme for unconstrained optimization Journal of Computational and Applied Mathematics, 234, pp. 3397-341

[3] Birigin, F. G. and Martinez, J. M., (2001), A Spectral Conjugate Gradient Method for Unconstrained Optimization, Applied Mathematics and Optimization, 43, 117-128.

[4] Dai, Y. and Yuan Y. (1999) ' A nonlinear conjugate gradients method with a strong global convergence property' SIAM J. Optim. 10, pp.177-182

[5] Fletcher, R. (1987) ' Practical Methods of Optimization (second edition). John Wiley and Sons, New York.

[6] Fletcher, R. and Reeves C. (1964) ' Function minimization by conjugate gradients ' Computer J. 7, pp.149-154.

[7] Hestenes, M. R. and Stiefel E. L. (1952) ' Method of conjugate gradients for solving linear systems' J. Research Nat. Standards 49, pp.409-436.

[8] Liu Y. and Storey C. (1991) ' Efficient generalized conjugate gradients algorithms ' Part 1 : Theory. J. Opti. Theory and Appli. 69, pp.129-137.

[9] Polak, E. and Ribiere, G. (1969) ' Note for Convergence Direction Conjugate, Revue Francaise Informant, Reserche. Opertionelle, 16, 3543.

[10] Wei Z., Tang C. and Li G. (2007) ' New conjugacy conditions and related new conjugate gradient methods for unconstraint optimizatio' J. of computation and Applied Mathematics, 202, pp.523-539.

[11] Wei Z., Mo L. and Lu S. (2011) ' Some global convergence properties of the Wei-Yao-Liu conjugate gradient with inexact line search ' J. Applied Mathematics and computation 217, pp.7132-7137.

[12] Kafaki S.B., Ghanbari R. and Amiri M.N., (2010), Two new conjugate gradient method based on modified secant equations, Journal of computational and applied Mathematics, 234, 1374-1386.

[13] Sakaiwa, N., Takano, M. and Yabe, H., (2003), Global Convergence Properties of New Nonlinear Conjugate Gradient Methods for Unconstrained Optimization, Department.

[14] Yasushi N., Hideaki I., (2011). Conjugate gradient methods using value of objective function for unconstrained optimization Optimization Letters, V.6, Issue 5, 941-955. 